\title{
Sunitinib Treatment for Metastatic Renal Cell Carcinoma in Patients with Von Hippel-Lindau Disease
}

\author{
Ho Cheol Kim, MD \\ Jung Su Lee, MD \\ Sang Hyung Kim, MD \\ Hoon Sub So, MD \\ Chang Yoon Woo, MD \\ Jae Lyun Lee, MD
}

Von Hippel-Lindau (VHL) disease is an autosomal dominant disease that produces a variety of tumors and cysts in the central nervous system and visceral organs, including renal cell carcinoma (RCC). RCC in patients with VHL disease does not frequently metastasize, therefore, the response to treatment and prognosis of metastatic RCC developed in patients with VHL disease has not been reported. Sunitinib is an oral, multitargeted receptor tyrosine kinase inhibitor with antiangiogenic and antitumor activity. Here, we report on four patients with metastatic RCC in VHL disease who received sunitinib and achieved partial responses that have lasted for a prolonged period of time.

Correspondence: Jae Lyun Lee, MD, PhD Department of Oncology, Asan Medical Center, University of Ulsan College of Medicine, 88 Olympic-ro 43-gil, Songpa-gu, Seoul 138-736, Korea

Tel: 82-2-3010-3210

Fax: 82-2-3010-6961

E-mail: jaelyun@amc.seoul.kr

Received November 27, 2012

Accepted January 3, 2013

\author{
Key words \\ Von Hippel-Lindau disease, Neoplasm metastasis, \\ Renal cell carcinoma, Sunitinib
}

\section{Introduction}

Von Hippel-Lindau (VHL) disease is a rare condition which is inherited in an autosomal dominant pattern. The $V H L$ gene is located on the short arm of chromosome 3 and is caused by germline mutations. This condition causes development of benign and malignant tumors in the central nervous system and the internal organs; tumors caused by VHL mutation include hemangioblastomas in the cerebel- lum, spinal cord, brain stem and retina, renal cell carcinoma (RCC), pheochromocytoma, and pancreatic carcinoma [1].

The incidence of RCC has been increasing and metastasis is known to occur in approximately $30 \%$ of tumors of this type [2]. Previously, metastatic RCC patients were treated with immunotherapy using interferon alpha or interleukin2 [3], however, more recent treatments include vascular endothelial growth factor (VEGF) monoclonal antibodies, tyrosine kinase inhibitors (TKI), and mammalian target of rapamycin (mTOR) inhibitors [4-6]. Approximately $40 \%$ to 
$70 \%$ of patients with VHL disease also have RCC, whereas only $25 \%$ are known to have metastatic RCC [7]. However, the response to VEGF TKI treatment, and the progression and prognosis of metastatic RCC in VHL patients are not yet well known.

Here, we report on four patients with metastatic RCC complicating VHL disease treated with sunitinib (Sutent, Pfizer, New York, NY); these cases suggest that metastatic RCC associated with VHL is highly responsive to VEGF TKI for a prolonged period of time, and benign cysts or tumors could be also controlled with VEGF TKIs.

\section{Case Reports}

\section{Case 1}

A 42-year-old female was found to have masses in both kidneys four years ago and was transferred to Asan Medical Center. Her family history included VHL disease in her father and her older sister. She also had hemangioblastoma in the cerebellum, which was removed 16 years ago. Abdominal computed tomography (CT) scan performed at this hospital showed masses in both kidneys which were suspected to be RCC; thus, the patient underwent partial nephrectomy of the left kidney. Two months later, the patient underwent radical nephrectomy of the right kidney. Pathology of the samples revealed clear cell RCC in both kidneys. The patient developed local recurrent RCC in the left kidney two years after nephrectomy and was subsequently treated by radiofrequency ablation. The patient then developed multiple metastases in the lungs and was transferred to the oncology department.

Chest CT subsequently confirmed multiple metastases in both lung fields and the patient was diagnosed with lung metastases of RCC and started treatment of sunitinib (50 mg for two weeks, followed by a one-week rest period). The metastatic tumors were found to have regressed following sunitinib administration and reached partial response according to the Response Evaluation Criteria in Solid Tumors (RECIST) criteria (Fig. 1). After administration of sunitinib for 16 months, the patient suffered hand and foot neuropathy (grade 2), and wanted a dose reduction; we decided on a dose reduction of sunitinib ( $25 \mathrm{mg}$ for two weeks, followed by a one-week rest period). After that, this patient became tolerable to treatment. This partial response has been maintained at a steady state for three years and three months with sunitinib administration. No changes in
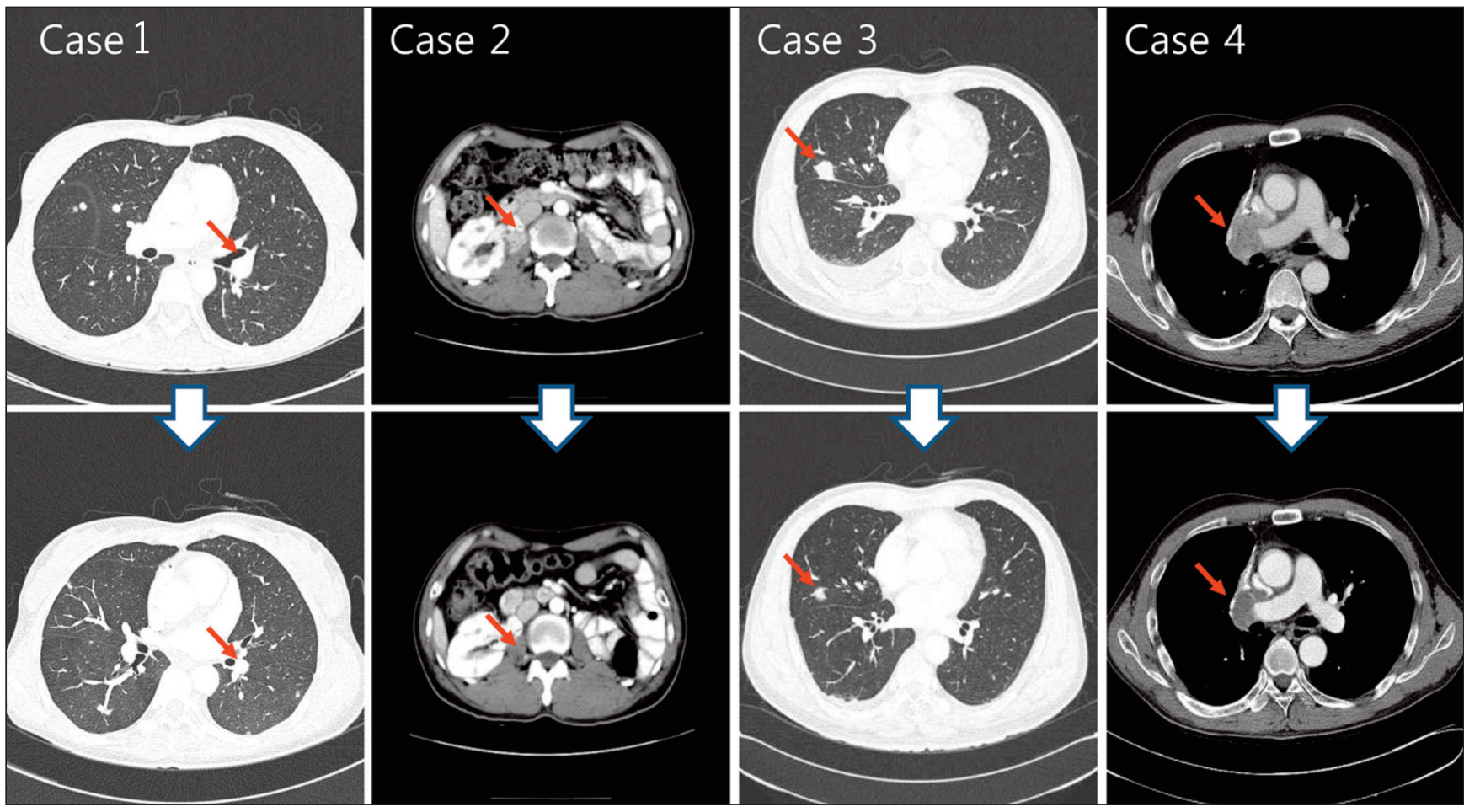

Fig. 1. Response of metastatic renal cell carcinoma developed in patients in Von Hippel-Lindau disease to sunitinib. Selected target lesions showed favorable response. 

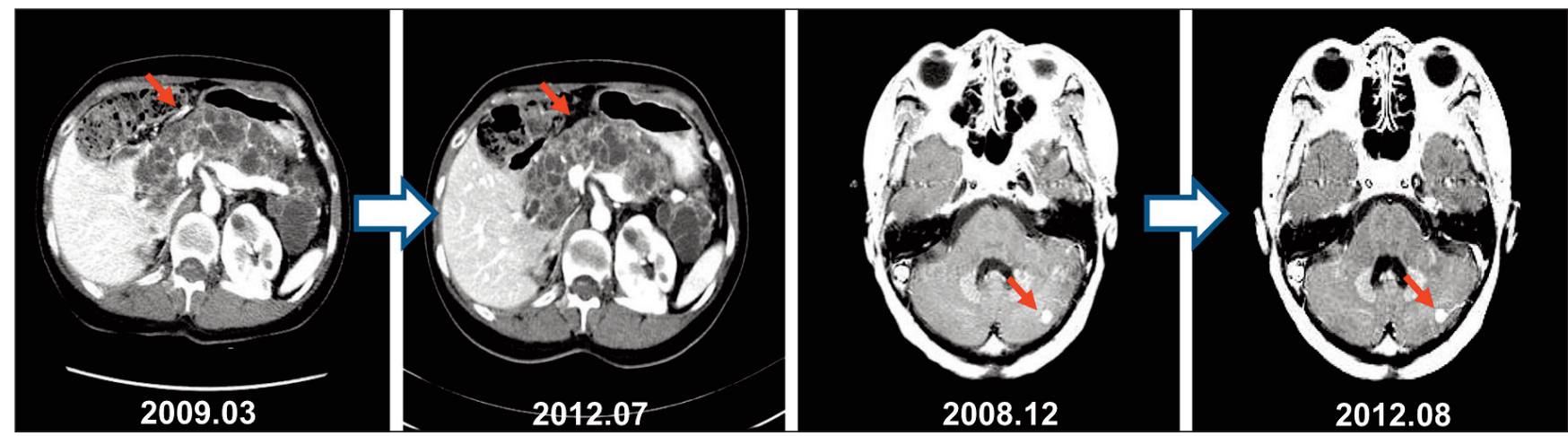

Fig. 2. No change in size of cerebellar hemangioblastoma and pancreatic cysts during sunitinib treatment.

pancreatic mass lesions or hemangioblastoma of the cerebellum during administration of sunitinib were observed during that period of time (Fig. 2). After that, a chest CT scan showed disease progression in the lung; therefore, the patient's treatment was changed to everolimus (Afinitor, Novartis, Basel, Switzerland). Chest CT at two months of treatment with everolimus showed progression of the disease, therefore, the medication was changed again to sorafenib (Nexavar, Bayer, Leverkusen, Germany). The patient also achieved partial response to sorafenib and is currently receiving treatment with sorafenib for five months.

\section{Case 2}

A 45-year-old male patient was diagnosed with bilateral RCC five years ago and subsequently underwent partial left nephrectomy and radical right nephrectomy. Pathology of the samples revealed clear cell RCC in both kidneys. At this time, the patient was found to have multiple pancreatic cysts and was diagnosed with RCC and VHL disease. At three months post-operative follow-up, the patient was admitted to the hospital with right flank pain. Abdominal CT scan showed a recurrent RCC measuring $2.5 \mathrm{~cm}$ in size in the right psoas muscle adjoining the partially nephrectomized kidney. The patient started on sunitinib treatment $(50 \mathrm{mg}$, four-week treatment with a two-week rest period). Treatment with sunitinib resulted in regression of the tumor, and at six months, the patient had achieved a partial response (Fig. 1). At 21 months, the patient is still undergoing treatment with sunitinib and is being followed up.

\section{Case 3}

A 49-year-old male patient was diagnosed with RCC in the right kidney five years ago and underwent radical nephrectomy of that kidney. Pathology of the sample revealed clear cell RCC. At that time, the patient was also found to have hemangioblastoma in the right retina and the cerebellum and was diagnosed with VHL disease. On surveillance chest CT, multiple lung and pleural metastases were found. The patient was treated with sunitinib $(50 \mathrm{mg}$, four-week treatment with a two-week rest period) and achieved partial response according to RECIST criteria four months after treatment with sunitinib (Fig. 1), which lasted four years and three months. Follow-up chest CT showed disease progression and the patient's medication was changed to everolimus. After two months of treatment with everolimus, response studies showed further disease progression and the patient is now receiving supportive care without further chemotherapy due to poor general condition.

\section{Case 4}

A 44-year-old male patient presented to the hospital with left flank pain four years ago. Abdominal CT scan showed bilateral RCC and the patient subsequently received left radical nephrectomy and right partial nephrectomy. Pathology of the samples showed clear cell RCC in both kidneys. His older sister had been diagnosed with VHL disease and the patient also had several pancreatic cysts and VHL gene mutation (3p26-p25) resulting in a diagnosis of VHL disease. Three years prior to admission, the patient was found to have lung metastases in the right middle and lower lobes and underwent wedge resection of the right middle and lower lobes. Chest CT scan performed during post-operative follow up showed enlarged right hilar lymph nodes along with a metastatic mass invading into the superior vena cava. The patient was started on sunitinib treatment $(50 \mathrm{mg}$, fourweek treatment with a two-week withdrawal period). After three months of treatment with sunitinib, the patient achieved partial response according to RECIST criteria (Fig. 1). Following treatment with sunitinib for one year and 11 months, the patient's abdominal and chest CT scan showed disease progression and the treatment was changed 


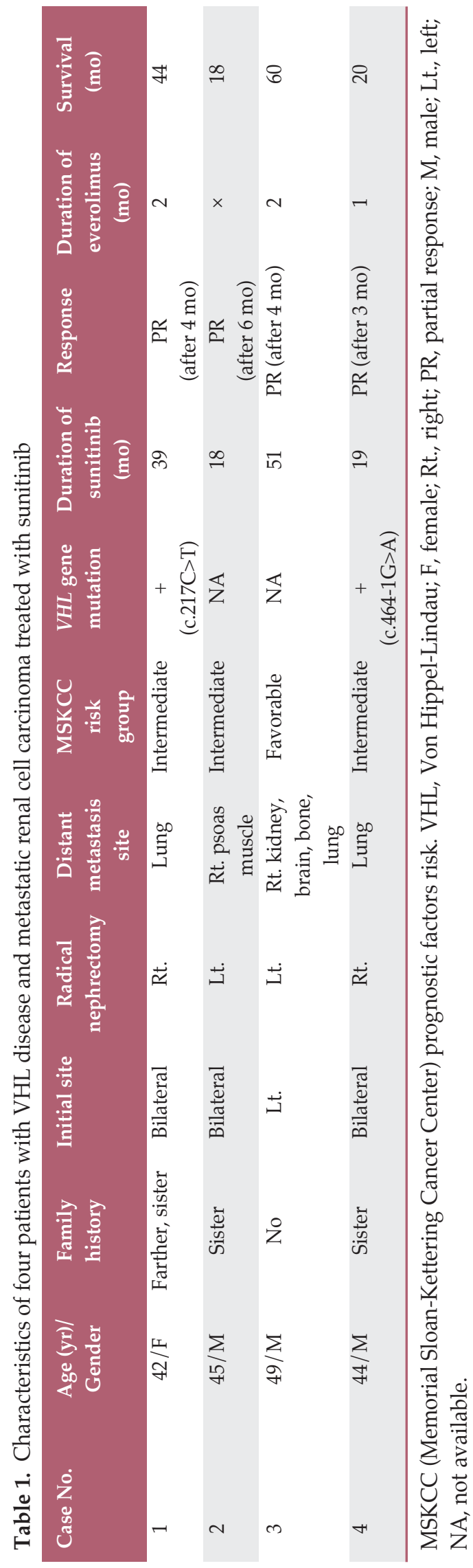

to everolimus. The patient is currently receiving treatment with everolimus for two months (Table 1).

\section{Discussion}

The diagnosis of VHL disease is made by the presence of at least one of the following: 1) hemangioblastomas of cerebellum, cerebrum, or medulla, 2) retinal hemangioblastomas, 3) multiple renal cysts or adenoma or RCC, 4) pancreatic cyst or adenoma or islet cell tumor, 5) pheochromocytoma, or 6) epididymis cystadenoma; along with a family history of the disease. If there is no family history of VHL disease, the diagnosis can be made if the patient has at least two of the conditions listed above [1].

Development of RCC occurs in $40 \%$ to $70 \%$ of patients with VHL disease. However, fewer than $25 \%$ of RCC patients with VHL disease have metastatic RCC [7]. The response to treatment, disease course, and prognosis of metastatic RCC complicating VHL disease have not yet been thoroughly addressed, particularly in the era of targeted therapy.

The VHL gene, which is known as a tumor suppressor gene and is related to protein formation, plays a role in angiogenesis and formation of extracellular matrix. The VHL protein participates in ubiquitin protein degradation of hypoxia inducible factor (HIF) in the case of VHL protein combined with HIF. As in VHL disease, mutations in the VHL gene cause disruption in HIF degradation and induce over-expression of proteins, including VEGF (the target protein), platelet derived growth factor (PDGF), and transforming growth factor. This phenomenon could be the reason for an excessive angiogenesis and cellular proliferation [1].

According to one report, use of VEGF receptor TKI therapy in localized, non-metastatic, RCC associated with VHL disease led to partial response [8], however, the response and outcomes in patients with advanced, metastatic RCC has not yet been reported.

Sunitinib, TKI of VEGF and PDGF receptors, has been proven effective for treatment of metastatic RCC. A phase III clinical study comparing the efficacy of sunitinib with interferon alpha in metastatic RCC reported a clinical benefit in terms of better progression-free survival, 11 months, and a response rate of $31 \%$ in patients treated with sunitinib [4]. A retrospective study conducted in Korean patients reported a response rate of $43 \%$ and time to disease progression of 11.8 months, proving its efficacy in Asian patients [9].

It is interesting that all four of the patients in this case series achieved partial response to sunitinib, which lasted for a prolonged period of time; two to five times of median progression-free survival reported in a phase III study. This 
finding suggests that sunitinib is much more effective in RCC complicating patients with VHL disease compared to sporadic RCCs not associated with VHL disease. Although one patient had dose modification due to side effect and all patients experienced several adverse events (e.g., skin rash, and foot syndrome, neuropathy, stomatitis, mucositis, etc.), none of the patients developed severe adverse events (above grade 3 ) and all patients showed tolerability during the treatment period.

The use of mTOR inhibitors such as everolimus in patients with VHL disease led to disease progression within the first two months of treatment in two patients. Although only a limited number of patients were included in this series, our experience also raises a question regarding the efficacy of mTOR inhibitors in patients with VHL disease after failure of VEGF TKI. In the first case, the patient achieved partial response with sorafenib after her disease showed progression despite treatment with sunitinib and everolimus, suggesting that the sensitivity to VEGF TKI can be recovered after stopping VEGF TKI treatment and this may be accentuated in patients with VHL disease $[10,11]$.

This case study also showed that there were no significant changes in the sizes of cystic lesions in the pancreas and hemangioblastomas in cerebellum with sunitinib treatment. Approximately $44 \%$ of hemangioblastomas in cerebellum of VHL patients show a gradual increase in size with time [12], as with pancreatic lesions, $48 \%$ of lesions also tend to increase in size [13]. The fact that the size of lesions of the pancreas and cerebellum did not increase during sunitinib treatment suggests that these lesions may also respond to sunitinib treatment; this would need to be evaluated further in the future.

In conclusion, metastatic RCC associated with VHL is highly responsive to VEGF TKI for a prolonged period of time, and benign cysts or tumors associated with VHL disease could also be controlled with VEGF TKIs.

\section{Conflicts of Interest}

Conflict of interest relevant to this article was not reported.

\section{References}

1. Lonser RR, Glenn GM, Walther M, Chew EY, Libutti SK, Linehan WM, et al. von Hippel-Lindau disease. Lancet. 2003;361: 2059-67.

2. Motzer RJ, Bander NH, Nanus DM. Renal-cell carcinoma. N Engl J Med. 1996;335:865-75.

3. Negrier S, Escudier B, Lasset C, Douillard JY, Savary J, Chevreau $C$, et al. Recombinant human interleukin-2, recombinant human interferon alfa-2a, or both in metastatic renalcell carcinoma. Groupe Francais d'Immunotherapie. N Engl J Med. 1998;338:1272-8.

4. Motzer RJ, Hutson TE, Tomczak P, Michaelson MD, Bukowski $\mathrm{RM}$, Rixe $\mathrm{O}$, et al. Sunitinib versus interferon alfa in metastatic renal-cell carcinoma. N Engl J Med. 2007;356:115-24.

5. Hudes G, Carducci M, Tomczak P, Dutcher J, Figlin R, Kapoor A, et al. Temsirolimus, interferon alfa, or both for advanced renal-cell carcinoma. N Engl J Med. 2007;356:2271-81.

6. Escudier B, Bellmunt J, Negrier S, Bajetta E, Melichar B, Bracarda $S$, et al. Phase III trial of bevacizumab plus interferon alfa$2 \mathrm{a}$ in patients with metastatic renal cell carcinoma (AVOREN): final analysis of overall survival. J Clin Oncol. 2010;28:214450.

7. Friedrich CA. Von Hippel-Lindau syndrome: a pleomorphic condition. Cancer. 1999;86(11 Suppl):2478-82.
8. Jonasch E, McCutcheon IE, Waguespack SG, Wen S, Davis DW, Smith LA, et al. Pilot trial of sunitinib therapy in patients with von Hippel-Lindau disease. Ann Oncol. 2011;22:2661-6.

9. Yoo C, Kim JE, Lee JL, Ahn JH, Lee DH, Lee JS, et al. The efficacy and safety of sunitinib in korean patients with advanced renal cell carcinoma: high incidence of toxicity leads to frequent dose reduction. Jpn J Clin Oncol. 2010;40:980-5.

10. Rini BI, Escudier B, Tomczak P, Kaprin A, Szczylik C, Hutson $\mathrm{TE}$, et al. Comparative effectiveness of axitinib versus sorafenib in advanced renal cell carcinoma (AXIS): a randomised phase 3 trial. Lancet. 2011;378:1931-9.

11. Zama IN, Hutson TE, Elson P, Cleary JM, Choueiri TK, Heng DY, et al. Sunitinib rechallenge in metastatic renal cell carcinoma patients. Cancer. 2010;116:5400-6.

12. Wanebo JE, Lonser RR, Glenn GM, Oldfield EH. The natural history of hemangioblastomas of the central nervous system in patients with von Hippel-Lindau disease. J Neurosurg. 2003;98:82-94.

13. Hammel PR, Vilgrain V, Terris B, Penfornis A, Sauvanet A, Correas JM, et al. Pancreatic involvement in von HippelLindau disease. The Groupe Francophone d'Etude de la Maladie de von Hippel-Lindau. Gastroenterology. 2000;119: 1087-95. 